\title{
Aufmerksamkeitsdefizit-Hyperaktivitätsstörung
}

\section{Auch Erwachsene mit ADHS beno̊tigen multimodale Therapie}

Berichte von Symposien der Pharmaindustrie
- Die Aufmerksamkeitsdefizit-Hyperaktivitätsstörung (ADHS) manifestiert sich zwar bereits in der Kindheit, persistiert aber bei einem Teil der Betroffenen bis in das Erwachsenenalter. Mit steigendem Alter ändert sich die Symptomatik, sie beeinträchtigt die Betroffenen dennoch in vielfältiger Weise im beruflichen wie im sozialen Leben.

\section{Symptome ändern sich}

Charakteristische Symptome der ADHS mit begleitenden Einschränkungen halten bei bis zu $70 \%$ der Betroffenen von der Kindheit ins Erwachsenenalter hinein an, berichtete Professor Martin Holtmann, Hamm. Allerdings vermindere sich im Altersverlauf die Trias aus motorischer Unruhe, Defiziten in der Konzentration und erhöhter Impulsivität. Stattdessen berichteten junge Erwachsene mit ADHS fast regelhaft von mangelnder Alltagsorganisation, ineffizienter Arbeitsweise, schlechter Impulskontrolle und emotionaler Labilität. Als ein Beispiel für Probleme jenseits der Kernsymptome nannte Holtmann die im Vergleich zu Kontrollpersonen bis zu 40-fach erhöhte Rate von Teenagerschwangerschaften unter Jugendlichen mit ADHS.

Die erfolgreiche Behandlung der ADHS im Erwachsenenalter setzt laut Professor Ernst-Wolfgang Rudolf
Retz, Homburg, eine gründliche diagnostische Abklärung voraus. Bei Verdacht auf ADHS sei eine besonders sorgfältige Abklärung der Frage nötig, ob in der Kindheit bereits Symptome der ADHS vorgelegen hätten. Retz rät zum Einsatz validierter ADHS-spezifischer Selbstund Fremdbeurteilungsverfahren, welche Befunddokumentation und Kontrolle des Behandlungsverlaufs erleichterten.

Erwachsene mit ADHS sollten eine multimodale Behandlung aus Psychoedukation, Verhaltens- und Pharmakotherapie erhalten, betonte Professor Michael Rösler, Homburg. Dabei gelte Methylphenidat weltweit als Mittel der ersten Wahl. Laut Metaanalysen sei Methylphenidat bei Erwachsenen mit ADHS Placebo signifikant überlegen. Zwar seien die Effektstärken im Vergleich zu Kindern und Jugendlichen geringer, es gebe aber Hinweise auf einen Dosiseffekt mit besseren Ergebnissen bei höheren Dosierungen. Seit Juli 2011 ist mit Medikinet adult $^{\star}$ erstmals ein Methylphenidat-Präparat zur Behandlung der adulten ADHS zugelassen und erstattungsfähig.

Dr. Gunter Freese

Satellitensymposium „ADHS bei Erwachsenen - Aktuelle Aspekte der Therapie“, DGPPN-Kongress, Berlin, 24.11.2011; Veranstalter: Medice

\section{Depression}

\section{Mit Schlafstabilisierung kann viel erreicht werden}

- Der Melatoninagonist und $5 \mathrm{HT}_{2 \mathrm{c}}$-Antagonist Agomelatin (Valdoxan ${ }^{\circledR}$ ) ist nicht nur antidepressiv wirksam, er kann auch die Schlafeffizienz von Patienten mit Depression deutlich bessern. Ebenso ist die bei Therapie mit SSRI mitunter auftretende Affektverflachung („Emotional Blunting“) unter Agomelatin deutlich seltener zu verzeichnen.

In seinem Vortrag „Monoaminerge und zirkadiane Therapiemechanismen - die Zeitmaschine in uns" betonte Professor Göran Hajak, Bamberg, die gute Wirkung von Agomelatin auf den Schlaf-Wach-Rhythmus von Patienten mit Depression, der sehr häufig gestört sei. Laut den Ergebnissen einer 2011 veröffentlichten multizentrischen, randomisierten Doppelblindstudie mit 138 Patienten mit Depression habe Agomelatin bei vergleichbar guter Wirksamkeit den Schlaf-WachRhythmus im Vergleich zu Escitalopram signifikant besser stabilisiert, so Hajak [1]. Dies sei etwa an der REM-Schlaf-Latenz abzulesen, die unter Behandlung mit Agomelatin im Vergleich zu Escitalopram deutlich geringer ausfiel.

Unter Therapie mit SSRI tritt bei etwa $25 \%$ der Patienten ein sogenanntes Emotional Blunting (eine emo- tionale Verflachung oder Distanziertheit) auf. Im Vergleich zu Escitalopram schnitt Agomelatin in einer ebenfalls 2011 veröffentlichten Studiensubgruppe [2] mit 66 Patienten mit Depression bezüglich der Affektverflachung deutlich besser ab. Nach 24 Wochen bejahten unter Agomelatin-Behandlung signifikant weniger Patienten die Aussagen „Meinen Emotionen fehlt Intensität“ und „Dinge, die mich vor meiner Krankheit beschäftigten, erscheinen mir nicht mehr wichtig“ (20\% bzw. $18 \%$ ) als unter Escitalopram (58\% bzw. $42 \%$ ). Frühere Studien mit deutlich höheren Fallzahlen hatten bereits die antidepressive Wirksamkeit im Vergleich zu Placebo [3] beziehungsweise gegenüber SSRI und SNRI [4] gezeigt.

Heike Grosse

1. Quera-Salva MA et al. International Clinical Psychopharmacology: 2011; 26 (5): 252-62

2. Corruble E et al. Europ Psychiatry 2011; 26 (Suppl 1): P02-24

3. Demyttenaere K Eur Neuropsychopharmacol 2011; (Suppl 4): 703-9

4. Kasper S et al. J Clin Psychiatry 2010; 71 (2): 109-20

Satellitensymposium „Spektrum Depression: Differenzierung von Diagnostik und Therapie", DGPPN-Kongress, Berlin, 24.11.2011, Veranstalter: Servier Deutschland 